Technical Note

\title{
Monotonic non-linear transformations as a tool to investigate age-related effects on brain white matter integrity: A Box-Cox investigation
}

\author{
Maria Morozova ${ }^{\mathrm{a}}$, Karl Koschutnig a,b, Elise Klein ${ }^{\mathrm{c}}$, Guilherme Wood ${ }^{\mathrm{a}, \mathrm{b}, *}$ \\ a Department of Psychology, Karl-Franzens-University of Graz, Graz, Austria \\ b Biotechmed, Graz, Austria \\ c Knowledge Media Research Center Tuebingen, Tuebingen, Germany
}

\section{A R T I C L E I N F O}

\section{Article history:}

Received 17 February 2015

Accepted 1 August 2015

Available online 8 August 2015

\begin{abstract}
A B S T R A C T
Non-linear effects of age on white matter integrity are ubiquitous in the brain and indicate that these effects are more pronounced in certain brain regions at specific ages. Box-Cox analysis is a technique to increase the loglikelihood of linear relationships between variables by means of monotonic non-linear transformations. Here we employ Box-Cox transformations to flexibly and parsimoniously determine the degree of non-linearity of age-related effects on white matter integrity by means of model comparisons using a voxel-wise approach. Analysis of white matter integrity in a sample of adults between 20 and 89 years of age $(n=88)$ revealed that considerable portions of the white matter in the corpus callosum, cerebellum, pallidum, brainstem, superior occipitofrontal fascicle and optic radiation show non-linear effects of age. Global analyses revealed an increase in the average non-linearity from fractional anisotropy to radial diffusivity, axial diffusivity, and mean diffusivity. These results suggest that Box-Cox transformations are a useful and flexible tool to investigate more complex nonlinear effects of age on white matter integrity and extend the functionality of the Box-Cox analysis in neuroimaging.
\end{abstract}

(c) 2015 Elsevier Inc. All rights reserved.

\section{Introduction}

Effects of aging on white matter integrity are pronounced and have been well-documented in the literature (Abe et al., 2008; Hsu et al., 2008; Nusbaum et al., 2001; Pfefferbaum et al., 2000; Salat et al., 2005; Sullivan et al., 2001). Fractional anisotropy (FA) tends to decrease in most brain regions while mean diffusivity (MD) and radial diffusivity (RD) tend to increase with advancing age. Axial diffusivity (AD), however, shows a region-dependent behavior; some regions showing increased $\mathrm{AD}$ in function of age, and some showing decreased AD. Although the exact mechanisms driving these changes in diffusivity measures and FA are still not completely understood, it is generally agreed that the changes in diffusivity and FA indicate an age-related loss of fiber structure and mass in the white matter of the brain (Gong et al., 2014; Lockhart and DeCarli, 2014).

The shape of the relation between white matter integrity and age is not the same across different brain regions or different age groups. While in some regions changes in white matter integrity seem to change at a relatively constant pace across different age intervals, there are various examples in the literature of a non-linear relationship between diffusivity and FA (Michielse et al., 2010; Westlye et al., 2010). Moreover,

\footnotetext{
* Corresponding author at: Department of Psychology, Universitätsplatz 2, 8010 Graz, Austria. Fax: +433163809829.

E-mail address: guilherme.wood@uni-graz.at (G. Wood).
}

different parameters of diffusivity and FA also can show a specific behavior (Lebel et al., 2010). Several methods have been employed to investigate more complex associations between age and white matter integrity. One of them is to define regions or fiber tracts of interest or to parcellate the brain regions (Lebel et al., 2010; Pfefferbaum et al., 2013) and examine the impact of age in each one of them. Another one is to fit higherorder polynomials to diffusion values at a voxel-wise basis (Hsu et al., 2008). Generally, several methods are combined to provide information on more global and more local patterns of age-related changes in diffusivity and FA (Hsu et al., 2010; Westlye et al., 2010).

Higher-order polynomials are a useful and flexible tool to quantify non-linear properties of the association between variables (Hsu et al., 2010; Pfefferbaum et al., 2013; Sala et al., 2012; Westlye et al., 2010). Higher-order polynomials have the property to characterize nonmonotonic effects of age with the help of only a few model parameters describing transformations of the independent variables typically according to a priori integer exponents (i.e., quadratic and cubic terms). The employment of higher-order polynomials may have some limitations as well (Fjell et al., 2010).

First, consider a white matter structure in which the true relation between age and white matter integrity is a fourth-order polynomial: in this case, a third-order polynomial fitted to age will probably still convey significant results although the true degree of non-linearity is more extreme than the statistical model was specified to describe. Accordingly, if the correct exponent were 0.5 , a linear model would still 
show reasonable fit, although one may agree that it is only a coarse approximation to the correct shape of the age-related effect.

Second, the ability to model non-monotonicity may be not always an advantage when evaluating age-related effects in adult populations. Inspection of scatterplots depicting age vs. white matter integrity in the literature is illustrative in this respect: Usually, the non-monotonic agerelated effects are produced by the trajectory of early development occurring before individuals reach adult age (Fjell et al., 2010; Pfefferbaum et al., 2013; Sala et al., 2012; Westlye et al., 2010). For this reason, one may argue that evidence for non-monotonic age-related effects in adult life is limited at best, and that the ability of monotonic non-linear models to describe age-related effects in adults should be assessed.

Finally, under certain circumstances the researcher may prefer for reasons of parsimony and straightforwardness of interpretability not to decompose the effect of age into different terms age $+a g e^{2}+a g e^{3}$ but rather to preserve its natural scale. When this is the case, the dependent variable should be transformed.

The Box-Cox analysis (Box and Cox, 1964) is a technique to simplify statistical models by means of monotonic transformations of the dependent variable with several applications in neuroimaging, particularly regarding the Gaussian field based statistical models (Miranda et al., 2013; Pajevic, 2011; Ziegler et al., 2014).

As we will show in the following, Box-Cox transformations may help to overcome the limitations of higher-order polynomial regression enumerated above. First, Box-Cox transformations offer a straightforward way to determine the degree of nonlinearity by means of a $\lambda$ exponent, which can assume any real value and account flexibly and accurately for different degrees of non-linearity in the data. Second, BoxCox transformations are monotonic. Third, Box-Cox transformations are usually applied to the dependent variable, so that the interpretability of the effect of the predictor age is very good. The Box-Cox analysis performs transformations of data to obtain a coefficient $\lambda$ which can be interpreted as the degree of non-linear bias observed when computing the linear association between variables. The dependent variable $y$ is transformed in a monotonic and non-linear way as described in Eq. (1).

$y^{(\lambda)}=\left\{\begin{array}{l}\frac{y^{\lambda}-1}{\lambda} \lambda \neq 0 \\ \underset{\log (y)}{\lambda} \lambda=0\end{array}\right.$

According to this definition, only when the dependent variable $y$ is $>0$ the $\lambda$-exponent can be estimated (Box and Cox, 1964). When dealing with diffusivity or FA data, this is not an important limitation since one is interested mainly in brain regions showing some degree of diffusivity and FA, which is always larger than zero. Whenever the Box-Cox transformation can be calculated, one has a simple metric to express nonlinear associations between diffusivity and FA measurements and age in the brain.

A $\lambda$-exponent of 1 reveals that the association is linear and that no transformation of data is necessary. In turn, the farther away the $\lambda$ exponents are from 1 , the higher is the degree of non-linearity observed. When considering the relative impact of age, four main cases of nonlinearity can be identified, namely, two classes of accelerating ageeffects and two classes of decelerating age effects. Accelerating ageeffects, i.e., much larger age-related changes in later years than in young adulthood are found in two cases, when the diffusivity or FA are positively associated with age and the $\lambda$-exponent is $<1$ or when they are negatively associated with age and the $\lambda$-exponent is $>1$ (Fig. 1). Accordingly, decelerating age-effects characterize larger agerelated changes in young adulthood than in later years. Decelerating age-effects are associated with the $\lambda$-exponent $>1$ when diffusivity or FA is positively associated with age and a $\lambda$-exponent $<1$, when they are negatively associated with age (Fig. 1 ).

The $\lambda$-exponent established by means of Box-Cox analyses yields the monotonic transformation of the dependent variable that produces largest log-likelihood for the prediction of a dependent variable by a set of predictors (Sakia, 1992). For these reasons, the slopes on age calculated after the transformation of the dependent variable have always the same sign and are associated with $\mathrm{R}^{2}$ values which are at least as high as for the non-transformed dependent variable, when no nonlinearity is present in a certain voxel and higher $\mathrm{R}^{2}$ values, when non-linearity is present. In the present study, Box-Cox transformations were employed to determine voxel-wise the degree of non-linearity of ageeffects. We employed a combination of robust regression analysis and the Box-Cox transformations to develop a straightforward metric to determine the pace of age-related changes and their characterization as linear, accelerating, or decelerating age effects.

\section{Methods}

Participants

The sample included 88 healthy adults between the ages 18 and 89 ( $\mathrm{M}=45.47, \mathrm{SD}=18.37,54$ female; Table 1$)$. Written informed consent
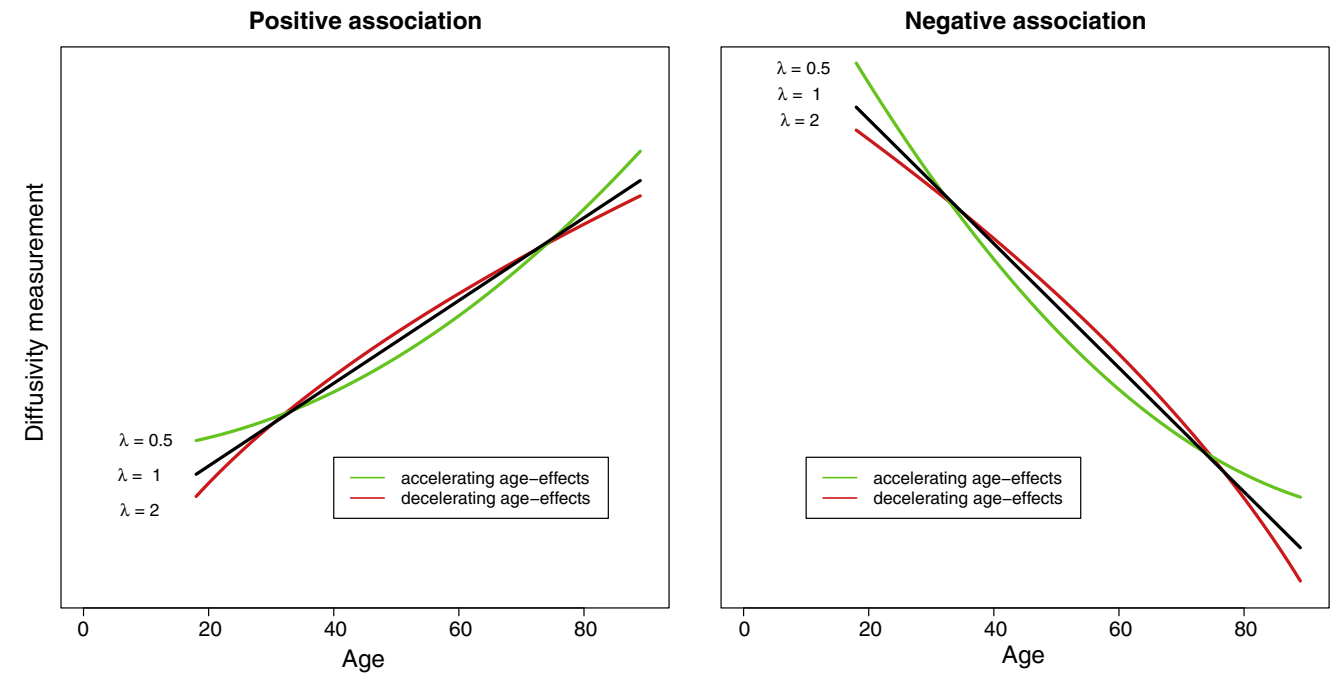

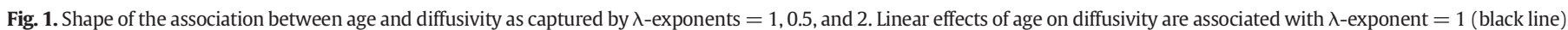

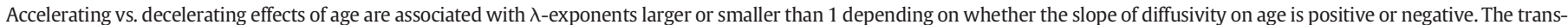

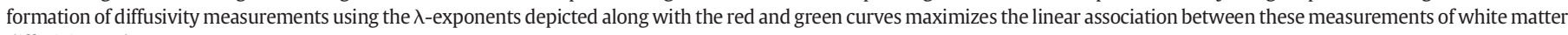
diffusivity and age. 
Table 1

Socio-demographic description of the sample.

\begin{tabular}{llll}
\hline $\begin{array}{l}\text { Age interval } \\
\text { (years) }\end{array}$ & $\begin{array}{l}\text { Mean age } \pm \text { SD } \\
\text { (years) }\end{array}$ & $\begin{array}{l}\text { Number of subjects } \\
\text { (males/females) }\end{array}$ & $\begin{array}{l}\text { Education } \\
(<12 \text { years/ } \geq 12 \text { years })\end{array}$ \\
\hline $18-39$ & $26.69 \pm 5.34$ & $36(17 / 19)$ & $22 / 14$ \\
$40-59$ & $49.04 \pm 6.31$ & $27(8 / 19)$ & $16 / 11$ \\
$60-89$ & $68.64 \pm 5.96$ & $25(9 / 16)$ & $13 / 12$ \\
\hline
\end{tabular}

was provided by all participants as well as a health-screening questionnaire. All participants were healthy and reported no history of chronic psychiatric or neurological illnesses, heart or brain surgery, and had normal or corrected to normal vision. Participants over 60 years were additionally screened for dementia using the Mini Mental State Examination (MMSE, Folstein et al., 1975), all scoring over 27 points and for depression using the General Depression-scale in German ("Allgemeine Depressionsskala"; Hautzinger et al., 2012) ensuring that they were not depressed and free from dementia. All participants received monetary reimbursement at the end of the testing. All but 10 participants were right-handed. Out of these 10 , three used their right hand to write. The study was approved by the local ethics committee and is in line with the Declaration of Helsinki.

\section{MRI acquisition}

Imaging data was acquired on a $3 \mathrm{~T}$ Siemens Skyra (Siemens Medical Systems, Erlangen, Germany) using a 32 channel head coil. Restraining foam pads were used to minimize head movement. For diffusion weighted images a single-shot echo planar imaging sequence was used. The parameters were: TR/TE/flip angle $=6600 \mathrm{~ms} / 95 \mathrm{~ms} / 90^{\circ}$, matrix size $=122 \times 122 \mathrm{~mm}$, FoV $=240 \mathrm{~mm}, 50$ transverse slices of $2 \mathrm{~mm}$ thickness measured, slice gap $=0.5 \mathrm{~mm}$, GRAPPA acceleration factor $=2.64$ diffusion sensitizing gradient directions applied (b-value $=1000 \mathrm{~s} / \mathrm{mm}^{2}$ ) and one non-diffusion weighted image ( $b$ value $=0 \mathrm{~s} / \mathrm{mm}^{2}$ ). A total acquisition time of $7 \mathrm{~min} 30 \mathrm{~s}$ was required. Structural T1 images were obtained using a MPRAGE sequence with the following parameters: $\mathrm{TR} / \mathrm{TE} / \mathrm{TI}=2530 / 2.07 / 900 \mathrm{~ms}$, flip angle $=9^{\circ}$, number of slices $=176$, slice thickness $=1 \mathrm{~mm}$, matrix $=256 \times 256$, and scan time $=6 \min 03 \mathrm{~s}$

\section{MRI data preprocessing}

First, b-values and b-vectors were extracted using MRIcron. The resulting images were preprocessed using the University of Oxford's Center for Functional Magnetic Resonance Imaging of the Brain (FMRIB) Software Library (FSL) in a standard multi-step procedure: (a) motion and eddy current correction, (b) removal of the skull and non-brain tissue using the Brain Extraction Tool (BET; Smith, 2002), and (c) voxel-wise calculation of diffusion tensors and computation of fractional anisotropy (FA), mean (MD), radial (RD), and axial diffusion (AD) maps using DTIfit. All of those steps are part of the FMRIB Diffusion Toolbox (FDT). The maps were used in the subsequent processing in Tract-Based Spatial Statistics (TBSS), which is also implemented in the FSL Toolbox. Each participant's FA volume was brought into a $1 \times 1 \times 1 \mathrm{~mm}^{3}$ common space (Montreal Neurological Institute space; MNI152) via the FMRIB58_FA template using FMRIB's nonlinear registration tool (FNIRT).

To ensure that each voxel used in the statistical analysis was really part of the white matter the subsequent procedure was followed. The T1-weighted images were registered to the b0-images of the same subject using a rigid body transformation in SPM12. The b0-image is the non-diffusion weighted image of the DWI data set, hence shows more signal. For each participant, the T1-image was segmented using SPM12 in native space (tissue types $=6$, sampling distance $=3$, segmented image voxel size $=1 \times 1 \times 1 \mathrm{~mm}^{3}$ ) resulting in individual white matter masks after binarization. These masks were applied to the FA-, MD-, RD-, and AD-images in native space. Thereafter, TBSS was used and the same voxelwise statistical analysis was carried out for the individual binary masks and the masked FA-, MD-, RD- and AD-images, by applying the nonlinear warps obtained from unmasked FA images. As the following statistical analysis is a voxel-wise one, we skipped the skeletonization step of TBSS. ${ }^{1}$ Using $\mathrm{R}$, a general mask was computed out of the individual masks now in MNI space, containing only voxels in which every participant presented white matter. This procedure left 230.079 voxels for statistical analysis.

\section{Box-Cox transformations and statistical analysis}

$\mathrm{FA}, \mathrm{MD}, \mathrm{RD}$, and $\mathrm{AD}$ images were masked using a white matter mask obtained from the mean of coregistered T1 images. Diffusion values were extracted from individual images. Only those FA, MD, RD, and $A D$ values larger than 0 observed consistently in every individual were considered in further analyses. A Box-Cox transformation analysis was employed to establish the value of the $\lambda$-exponent (Box and Cox, 1964) employed to maximize the log-likelihood for a regression model describing the effect of age on the (transformed) FA, MD, RD, and $A D$ values. The $\lambda$-exponent can be understood as an index of the degree of numerical compression necessary to linearize the relation between measurements of white matter integrity and a set of predictors. To perform the Box-Cox transformation analysis, different libraries of the software R were employed: "MASS" (Venables and Ripley, 2002) for the Box-Cox transformation and robust regression, "snowfall" for parallel computing (Knaus, 2013), "brainR" for display of imaging data (Muschelli, 2014), and "oro.nifti" (Whitcher et al., 2011) to read individual images in and out of R (CRAN, http://cran.r-project.org/). The $\lambda$ exponent maximizing the log-likelihood estimator was established in each voxel by means of successive approximations. Pilot analyses revealed that the numerical interval $[-6,6]$ was sufficient to capture the behavior of all measures of white matter integrity. After the pilot analyses, the search for the most adequate $\lambda$-exponent was set to be performed in the numerical interval between -6 and 6 in successive steps of 0.01 . The $\lambda$ value showing the highest log-likelihood for model fit for the model diffusivity measurement $<-$ age + sex + residuals was determined for each voxel individually and stored at its coordinates in a NIfTI image. The interpretation of $\lambda$ is the following: When the value of $\lambda$ is close to 1 , the relation with age + sex is considered to be

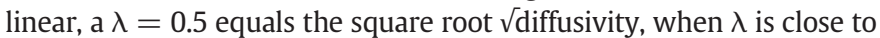
0 , the relation is logarithmic and so on (see Formula (1)). Generally, when the value is in the interval $(-6,1]$, the non-linear compression describes an acceleration of positive age-effects and a deceleration of negative age-effects in comparison to linear effects. When $\lambda$ values are in the interval $[1,6)$, the non-linear compression describes a deceleration of positive age-effects and an acceleration of negative age-effects. The Box-Cox analysis as performed combining the R libraries MASS, oro.NIfTI and snowball is not time-consuming and can be performed with considerable numerical accuracy within a few minutes of work on a standard PC even for a large number of participants and a large number of voxels per participant.

Non-linear transformations involving large exponents may lead to an inflation of the $\mathrm{R}^{2}$ due to the presence of outliers. To circumvent the negative impact of outliers in the estimation of the degree of nonlinearity of age-related effects, robust regression models were employed in all statistical calculations (Hampel et al., 1986). This is the standard procedure recommended to overcome problems with outliers which can be associated with Box-Cox transformations

\footnotetext{
1 No smoothing was applied to the diffusion images, as results obtained with different smoothing methods and different kernel sizes are heterogeneous (Assaf and Pasternak, 2008; Carmichael et al., 2013; Viswanath et al., 2012) and not always increase the validity of Gaussian assumptions (Jones et al., 2005).
} 
(Sakia, 1992). ${ }^{2}$ Since data are transformed monotonically in the BoxCox analysis, the interpretation of model fit is straightforward regarding the presence of deviations from linearity.

\section{Model evaluation and comparison}

To correct for multiple comparisons, $p$-values were thresholded to correct for false discovery rate (FDR; Benjamini and Yekutieli, 2001). A corrected threshold of $p<0.05$ and at least 80 voxels was considered statistically significant. Because both transformed and non-transformed models have the same number of degrees of freedom, the statistical comparison of both models was performed by means of the corrected Akaike Information Criterion (AICc, which adjusts the likelihood estimate for sample size). For the transformed and the non-transformed model, AICc values were calculated and normalized to obtain the Akaike weights $w_{i}$ (Burnham and Anderson, 2002) for each model in each voxel. The decision to consider the transformed model better than the non-transformed voxel in a given voxel was based on the confidence set of candidate models (analogous to a confidence interval for a mean estimate), which includes models with Akaike weights that are within $10 \%$ of the highest (Royall, 1997). When in a given voxel the Akaike weight $w_{\text {transformed model }}$ was at least 10 times larger than the $w_{\text {non-transformed model, }}$ we considered to have sufficient evidence to consider the transformed model to fit the age effects on diffusion data better than the non-transformed model.

\section{Results}

For the sake of straightforwardness, only voxel-wise analysis of data will be reported. Moreover, although sex-related effects have been included in the regression models, they will not be reported.

\section{Box-Cox analysis - global analysis of $\lambda$-exponents}

The regression slopes for age on DTI indices were calculated in each voxel. The number of voxels presenting either a positive or a negative slope on age is depicted in Table 2. The majority of voxels showed a decrease in FA and an increase in MD and RD in function of age. Moreover, approximately as many voxels showed either an increase or a decrease in AD. The $\lambda$-exponents were estimated in each voxel as well. Marginal distribution parameters are depicted in Table 2 separately for voxels showing either an increase or decrease in the diffusivity parameters and FA in function of age. Kernel density plots for the marginal distribution of $\lambda$-exponents are presented in Fig. 2. Voxels showing an increase as well as those showing a decrease in FA presented similar $\lambda$ exponents, which in average were in the interval between 0.5 and 1 . The $\lambda$-exponents of FA were slightly leptokurtic, what means that more voxels showed $\lambda$-exponents close to the average than would be expected from a Gaussian distribution. Together, these results suggest that the effect of age on FA only shows a low degree of non-linearity, irrespective of slopes being positive or negative. $\mathrm{RD}$ also shows a similar behavior, but the average $\lambda$-exponents between 0.22 and 0.75 suggest a stronger degree of non-linearity.

Interestingly, $\mathrm{MD}$ and $\mathrm{AD}$ have a more heterogeneous behavior. In voxels with a positive slope on age, the average $\lambda$-exponents of MD and $\mathrm{AD}$ were more strongly non-linear, since $\lambda<0$. However, in the voxels with a negative slope on age, the average $\lambda$-exponents of MD and $\mathrm{AD}$ were very close to linear as $\lambda$ was close to 1 . A more detailed inspection of the variance of $\lambda$-exponents of MD and AD reveals a heterogeneity at least two times as large as that observed in the $\lambda$-exponents

\footnotetext{
2 To ascertain that Box Cox transformations do not lead to capitalization on noise magnification, we run simulations of the form noise $\sim$ age + sex + residuals employing normal, lognormal and beta distributions to generate noise with different degrees of nonlinearity. Results of these simulations revealed that regardless of the non-linear transformation applied to data, statistical models evaluating the effect of age after transformation held to fair nominal significance levels i.e., alpha-error $=5 \%$ (see the S1 Support online materials for further details).
}

Table 2

Distribution of $\lambda$-exponents for diffusivity measurements and FA averaged across all voxels.

\begin{tabular}{llrrrlrr}
\hline & $\begin{array}{l}\text { Pos/neg } \\
\text { llope }\end{array}$ & $\begin{array}{l}\text { Number of } \\
\text { voxels }\end{array}$ & \multicolumn{1}{c}{$\begin{array}{l}\text { Median } \\
\text { FA }\end{array}$} & $\begin{array}{l}\text { Mean } \\
\lambda\end{array}$ & $\begin{array}{l}\text { Variance } \\
\lambda\end{array}$ & $\begin{array}{l}\text { Skew } \\
\lambda\end{array}$ & \multicolumn{2}{l}{$\begin{array}{l}\text { Kurtosis } \\
\lambda\end{array}$} \\
\hline \multirow{2}{*}{ MD } & 33,619 & 0.51 & 0.57 & 1.16 & 0.61 & 1.97 \\
& Neg & 196,460 & 0.75 & 0.76 & 0.91 & 0.28 & 1.71 \\
& Pos & 195,069 & -0.45 & -0.48 & 2.66 & 0.06 & -0.29 \\
RD & Neg & 33,847 & 0.85 & 0.69 & 2.16 & -0.47 & 0.41 \\
& Pos & 202,734 & 0.29 & 0.22 & 0.98 & -0.26 & -0.01 \\
AD & Neg & 21,740 & 0.84 & 0.75 & 0.75 & -0.43 & 0.39 \\
& Pos & 132,001 & -0.16 & -0.20 & 2.16 & -0.09 & -0.04 \\
& Neg & 97,821 & 0.62 & 0.59 & 1.73 & -0.11 & 0.08 \\
\hline
\end{tabular}

of FA and RD. In summary, the degree of non-linearity seems to increase from $\mathrm{FA}, \mathrm{RD}, \mathrm{AD}$ to $\mathrm{MD}$ and the variability of $\lambda$-exponents is smaller in $\mathrm{RD}$ and $\mathrm{FA}$ than in $\mathrm{AD}$ and $\mathrm{MD}$.

\section{Voxel-based analysis of age on DTI indices}

FDR-corrected $p$-values for the effect of age on different measurements of diffusion were calculated voxel-wise for non-transformed and Box-Cox transformed models separately using regression (Fig. 2). The Box-Cox transformed model produced a larger number of significant voxels than the non-transformed model for all diffusivity measurements and FA. With over 20,000 significant voxels, FA and RD showed the most pronounced age-related changes $\left(\mathrm{n}_{\mathrm{FAlin}}=21,094, \mathrm{n}_{\mathrm{FAbox}}=\right.$ $\left.21,540, \mathrm{n}_{\mathrm{RDlin}}=22,902, \mathrm{n}_{\mathrm{RDbox}}=23,572\right)$. A considerably smaller number of voxels presented age-related MD changes $\left(\mathrm{n}_{\text {lin }}=5830, \mathrm{n}_{\text {box }}=\right.$ 7135 ) and AD changes $\left(n_{\text {lin }}=2387, n_{\text {box }}=2503\right)$. The localization of neuroanatomical structures showing linear and non-linear age-related effects is reported below.

\section{Spatial localization of significant age-effects}

Clusters defined with the help of the Juelich Histological Atlas (Eickhoff et al., 2005, 2006, 2007) with an FDR corrected threshold of $p<0.05$ and $\mathrm{k}>80$ voxels are reported (Figs. 3 and 4 ). When the Juelich Histological Atlas could not localize one area, the Talairach Atlas (Lancaster et al., 2007) was used. Localization of clusters obtained for the non-transformed and the Box-Cox transformed model was comparable. For this reason, only the coordinates of the non-linear effects obtained from Box-Cox transformations will be described in detail, below.

\section{FA - Box-Cox transformed model}

The superior orbitofrontal fasciculus and the callosal body share the large cluster $(\mathrm{k}=16,437)$. Another cluster is shared by the body of CC and the left optic radiation ( $\mathrm{k}=377$ ), while two clusters can be assigned exclusively to the left $(\mathrm{k}=328, \mathrm{k}=84)$ and one to the right $(\mathrm{k}=151)$ optic radiation. Five clusters can be allocated in the CST, three in the left $(\mathrm{k}=452, \mathrm{k}=293, \mathrm{k}=130)$ and two in the right $(\mathrm{k}=226, \mathrm{k}=109)$ hemisphere. The left superior longitudinal fasciculus contains two clusters $(\mathrm{k}=283, \mathrm{k}=103)$, the brainstem one cluster $(\mathrm{k}=90)$ and the fornix, also one cluster $(\mathrm{k}=84)$. One cluster can be found in the left sub-lobar extra-nuclear white matter $(k=99)$.

\section{$M D$ - Box-Cox transformed model}

A similar pattern as for the linear model can be found for the BoxCox model. Seven out of 18 clusters are located in the body of CC, 4 in the left $(\mathrm{k}=818, \mathrm{k}=323, \mathrm{k}=100, \mathrm{k}=80)$ and three in the right hemisphere $(\mathrm{k}=626, \mathrm{k}=304, \mathrm{k}=86)$. Further 4 clusters can be assigned to the left CST ( $\mathrm{k}=590, \mathrm{k}=123, \mathrm{k}=121, \mathrm{k}=109)$ and one the right $(\mathrm{k}=644)$. Another cluster can be found in the right optic radiation $(\mathrm{k}=285)$, another in the right frontal $(\mathrm{k}=270)$, one in the left frontal white matter $(\mathrm{k}=274)$. Furthermore, one cluster can be assigned to the 
positive association

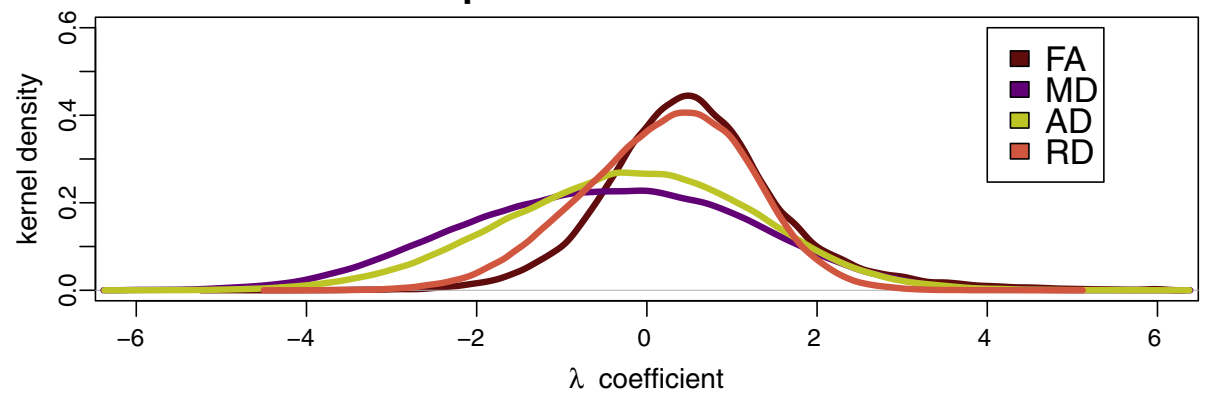

negative association

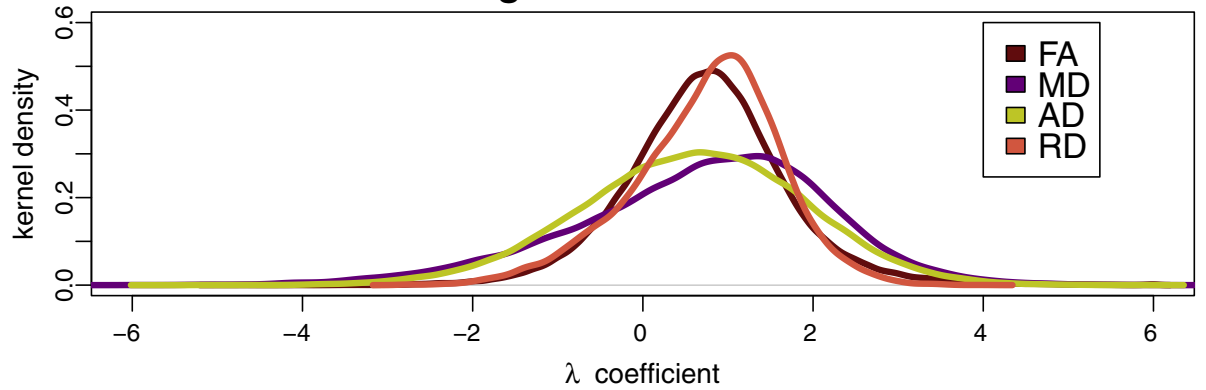

Fig. 2. Kernel density plots for the marginal distribution of $\lambda$-exponents of positive associations (above) and negative associations with age (below).

right sub-lobar extra nuclear white matter $(k=84)$, one to the left parietal $(\mathrm{k}=276)$ and the right temporal $(\mathrm{k}=81)$ white matter.

\section{$R D$ - Box-Cox transformed model}

The largest clusters are shared by the body of CC and the left superior orbitofrontal fasciculus $(\mathrm{k}=9633)$, the right cingulum $(\mathrm{k}=4556)$, the right CST $(\mathrm{k}=2809)$ and the right optic radiation $(\mathrm{k}=815)$. Two clusters can be exclusively allocated to the body of CC $(\mathrm{k}=1039, \mathrm{k}=288)$ and one to the left CST $(k=1200)$. Further two clusters can be found in the right frontal $(\mathrm{k}=255, \mathrm{k}=86)$ and two in the left parietal white $(\mathrm{k}=422, \mathrm{k}=132)$ matter.

\section{$A D-B o x-C o x$ transformed model}

5 clusters were found, four thereof can be found in the body of CC, one cluster in the left $(k=760)$, two clusters in the right $(k=587$, $\mathrm{k}=83$ ) hemisphere and one in between $(\mathrm{k}=101)$. The fifth cluster can be assigned to the CST $(\mathrm{k}=354)$.

\section{Model comparison: transformed $>$ non-transformed model}

Table 3 presents the distribution of $\lambda$-exponents where the transformed Box-Cox model fits better than the linear model. Strong nonlinear effects of age on FA, RD, AD, and MD were observed. There was a significantly better fit for the non-linear model for both, FA and AD in a substantial number of voxels $\left(\mathrm{k}_{\mathrm{FA}}=1749\right.$ and $\left.\mathrm{k}_{\mathrm{AD}}=1575\right)$. For $\mathrm{MD}$ and RD, respectively, an even larger number of voxels showed a significantly better fit for the non-linear model $\left(\mathrm{k}_{\mathrm{MD}}=5813\right.$ and $\mathrm{k}_{\mathrm{RD}}=$ 4365). The proportion of voxels showing either a positive or negative age-related effect was comparable to that observed in the marginal distribution in FA, MD, and RD: in all cases at least $80 \%$ of voxels showed either a decrease in FA or an increase in MD and RD. In contrast to the balanced ratio between positive and negative slopes observed in the marginal distribution of $\mathrm{AD}$ (Table 2), voxels with a positive slope on age predominated among those showing non-linear effects. For AD, over $80 \%$ of voxels revealing non-linear age related effects also showed a positive slope on age.

The $\lambda$-exponents in MD and AD were close to -3 and indicating considerably late onsets for accelerating positive age-effects as well as considerably early onsets for decelerating negative age-effects. Again, the degree of non-linearity in FA was lower than in $\mathrm{RD}, \mathrm{AD}$, and MD. Moreover, the variance of $\lambda$-exponents across voxels was generally larger in comparison to the marginal distribution (Table 2) for FA and AD, but comparable to MD and RD.

\section{Localization of non-linear age effects}

The spatial distribution of voxels showing significantly better model fit for the Box-Cox transformed model than for the non-transformed model was assessed with the help of the Juelich Histological Atlas (Eickhoff et al., 2005, 2006, 2007). Only clusters larger than $\mathrm{k}=80$ with $p$-values FDR-corrected to $5 \%$ are reported. A total of 22 different clusters of voxels were identified (Table 4 and Fig. 1 of Support online materials). Accelerating age-related effects have been observed in FA in the left cerebellar declive, in MD in the left and right callosal body, right optical radiation and the right sub-gyral white matter, in RD in the left and right callosal body and right optical radiation and, finally, in $\mathrm{AD}$ in the left superior occipito-frontal fascicle. Decelerating agerelated effects were observed in FA in the left pallidum and left superior occipito-frontal fascicle, and in both MD and RD in the left brainstem.

Scatterplots for the age-related effects are presented in Fig. 5A for all voxels pooled together and Fig. 5B for individual clusters. In Figs. 5A and $B$ transformed and non-transformed values are plotted in function of age and allow a view of the effects of Box-Cox transformation on diffusivity measurements and FA. A lowess fit curve for non-transformed data is also displayed as in previous work (e.g., Westlye et al., 2010). Transformed values tend to show a more linear association with age and the dispersion of data points is smaller than in the nontransformed data.

\section{Confidence intervals for $\lambda$-exponents in each neuroanatomical structure}

To further evaluate how individual neuroanatomical structures respond to non-linear age-related effects, 95\% lower and upper confidence intervals for individual voxels within each neuroanatomical structure were depicted in function of their $\lambda$-exponents (Fig. 6). MD, 


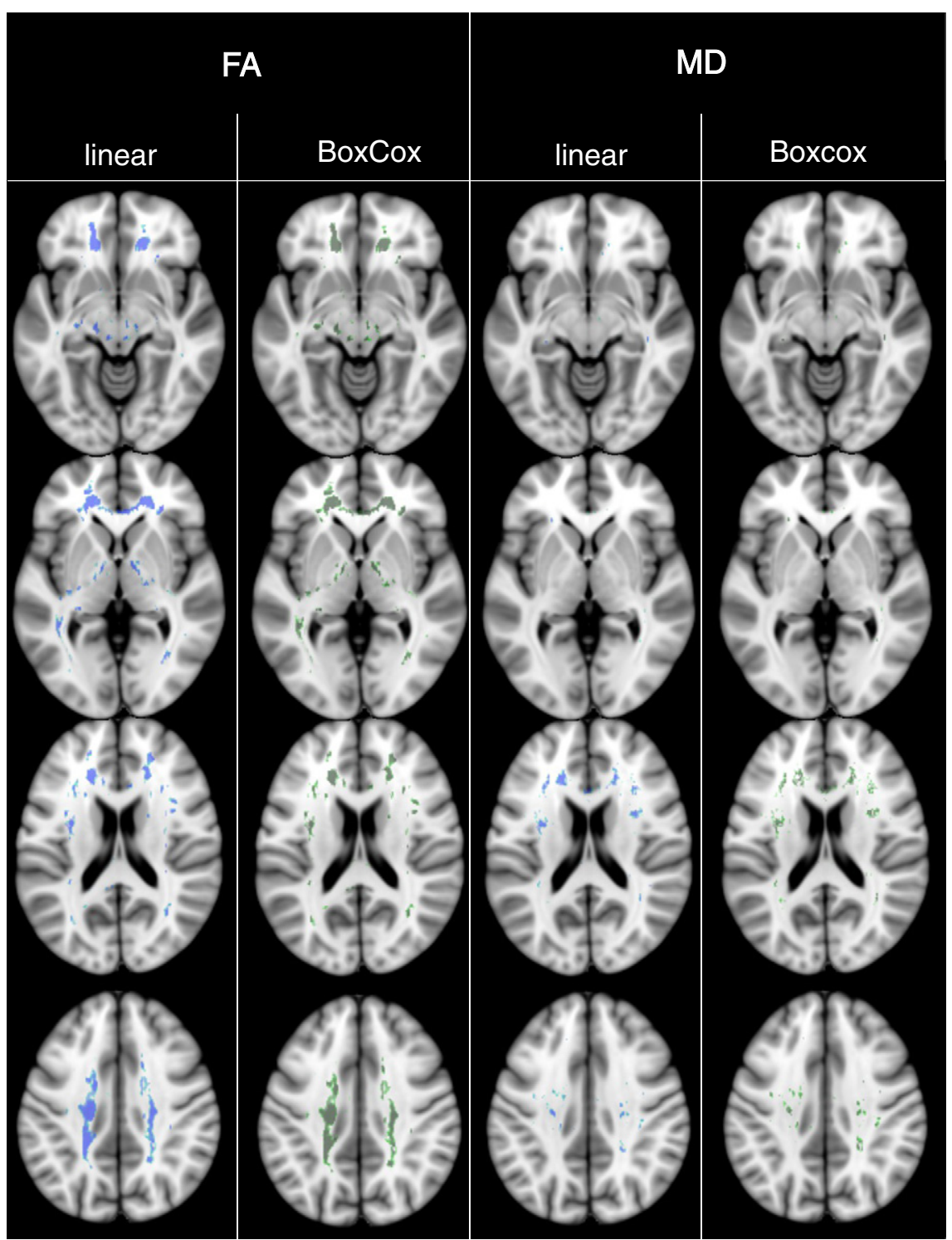

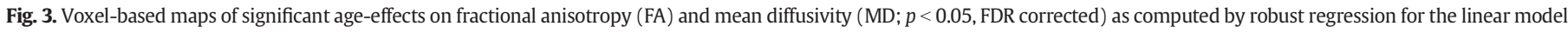
(blue) and the Box-Cox model (green).

AD and FA clusters show high heterogeneity while RD shows systematically a comparatively low heterogeneity in $\lambda$-exponents (Fig. 6).

Inspection of the first three plots of Fig. 6 reveals that although these clusters have a similar amount of voxels (Table 4) the heterogeneity of $\lambda$-exponents and confidence intervals for FA is larger in the left superior occipito-frontal fascicle than in the pallidum or in the cerebellar declive. MD showed large heterogeneity in the degree of non-linearity in the callosal body and optical radiation and slightly less variability in the subgyral white matter of the left prefrontal cortex and brainstem. A similar pattern was observed in smaller scale for RD in the same neuroanatomical structures. Finally, AD showed high variability in the degree of non-linearity in the callosal body and the left superior occipito-frontal fascicle. Generally these results point out that the heterogeneity of $\lambda$ exponents depends on the diffusion measurement at hand as well as on the neuroanatomical structure being examined.

\section{Discussion}

In the present study, we employed monotonic non-linear Box-Cox transformations (Box and Cox, 1964) to investigate effects of age on white matter diffusivity and FA. This approach may complement the more traditional methods based on the evaluation of higher-order polynomials to investigate non-linear effects of age and is in line with several other applications of Box-Cox transformations in neuroimaging (Miranda et al., 2013; Pajevic, 2011; Ziegler et al., 2014). In the BoxCox analysis, monotonic non-linear transformations were applied to diffusivity measurements and FA to determine which $\lambda$-exponent yields the highest log-likelihood when modeling the effects of age. The $\lambda$-exponent was calculated voxel-wise for FA, MD, RD, and $\mathrm{AD}$ and used to determine the degree of non-linearity of the effect of age. The majority of all voxels presented a $\lambda$-exponent close to 1 , meaning that the effect of age in these voxels seems to be largely linear. Nonetheless, a considerable number of voxels showed $\lambda$-exponents farther away from 1 , which is indicative of the presence of non-linear effects of age. Interestingly, a lower degree of non-linearity was observed in FA and $\mathrm{RD}$ and a higher one in AD and MD. Similar results were obtained when evaluating the variability of $\lambda$-exponents across voxels: FA and RD showed lower variance across voxels while the $\lambda$-exponents obtained for $\mathrm{AD}$ and $\mathrm{MD}$ revealed two times more variability. Inspection of the distribution of $\lambda$-exponents and the respective confidence intervals within clusters of voxels, in which strong non-linear age-related effects have been detected, revealed different degrees of heterogeneity in 


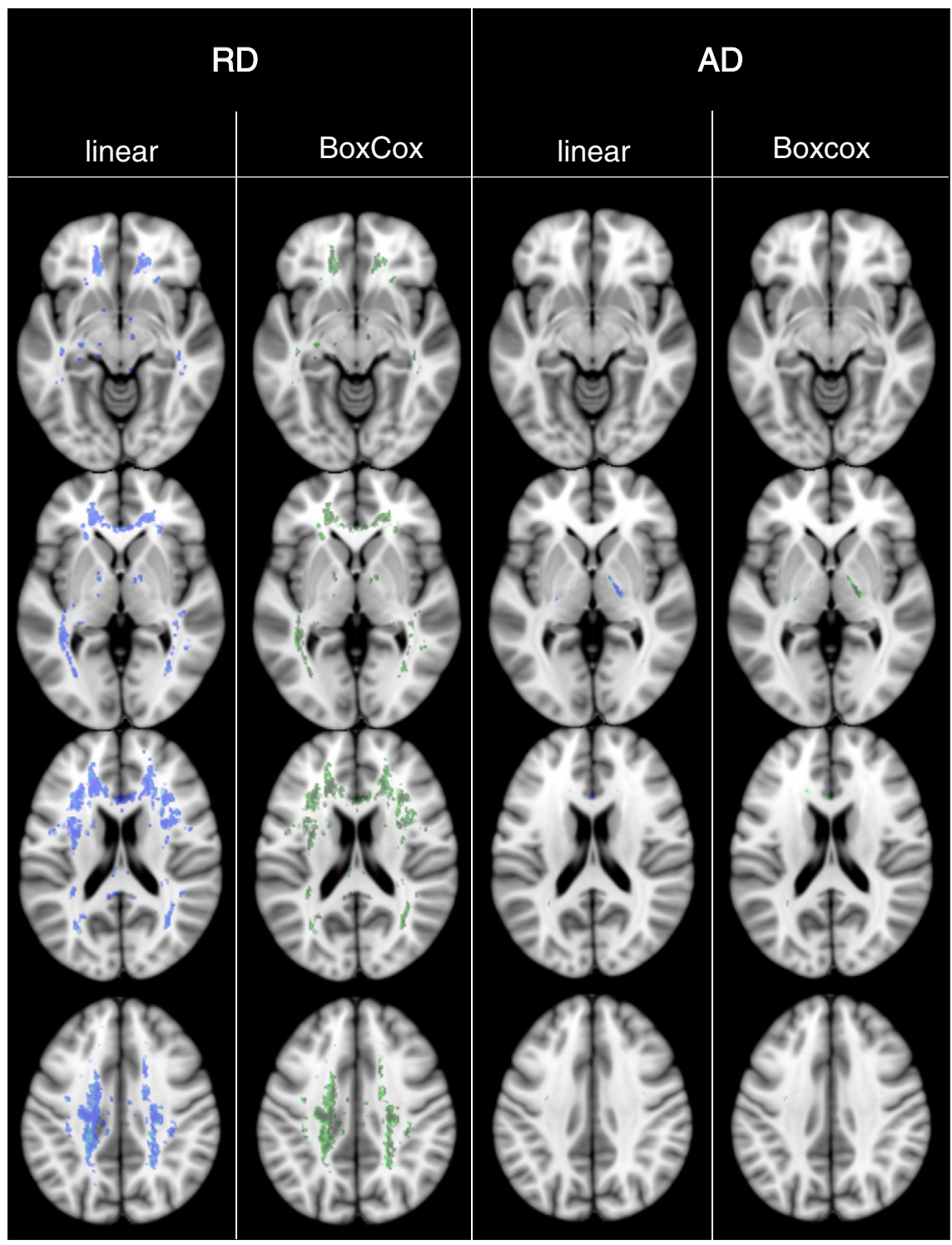

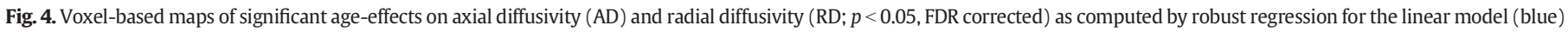
and the Box-Cox model (green).

$\lambda$-exponents. Some neuroanatomical regions presented very homogenous $\lambda$-exponents in single voxels while other ones presented very heterogeneous coefficients, which ranged from slightly non-linear to strong non-linearities. FA presents a distribution of $\lambda$-exponents similar to $\mathrm{AD}$ and $\mathrm{MD}$. Moreover, model comparisons revealed that the large majority of non-linear effects of age had a $\lambda$-exponent $<1$ regardless of the slope being positive or negative for all diffusivity measurements.

Table 3

Distribution of $\lambda$-exponents for diffusivity measurements and FA averaged across voxels where the Box-Cox model fits better than the linear model.

\begin{tabular}{llcllll}
\hline & $\begin{array}{l}\text { Pos/neg } \\
\text { Slope }\end{array}$ & $\begin{array}{l}\text { Number of } \\
\text { voxels }\end{array}$ & $\begin{array}{l}\text { Median } \\
\lambda\end{array}$ & $\begin{array}{l}\text { Mean } \\
\lambda\end{array}$ & $\begin{array}{l}\text { Varianz } \\
\lambda\end{array}$ & $\begin{array}{l}\text { Percentage of } \\
\text { significance }\end{array}$ \\
\hline FA & Pos & 183 & -0.93 & -0.80 & 1.17 & 0.5 \\
& Neg & 1566 & -0.76 & -0.43 & 2.58 & 0.8 \\
MD & Pos & 5477 & -3.05 & -2.94 & 1.73 & 2.8 \\
& Neg & 336 & -1.96 & -1.77 & 2.26 & 1.0 \\
RD & Pos & 4158 & -1.52 & -1.43 & 0.91 & 2.0 \\
& Neg & 207 & -1.01 & -0.99 & 0.52 & 1.0 \\
AD & Pos & 1382 & -3.04 & -3.01 & 1.25 & 1.0 \\
& Neg & 293 & -2.06 & -1.22 & 5.94 & 0.2
\end{tabular}

For FA on the other hand, one cluster was found showing a negative slope and $\lambda$-exponents $>1$. Finally, a large number of neuroanatomical structures presented accelerating non-linear age-related effects, whereas a smaller set of other regions showed decelerating non-linear agerelated effects.

These results replicate and extend previous findings obtained with higher-order polynomial regression models. Moreover, the localization of non-linear age-related changes in the corpus callosum, globus pallidus, left brainstem, and optic radiation is in line with the literature and will be discussed in more detail below.

The distribution of $\lambda$-exponents for effects of age on white matter integrity

The distribution of $\lambda$-exponents across voxels for FA, MD, RD, and AD was confined to the interval between -6 and 6 and showed a clear central tendency. More importantly, the large majority of voxels showed $\lambda$-exponents in the interval between -4 and 4 . As shown in Fig. 6 , the confidence intervals for these estimates involved in some cases values even below -6 . These results indicate that the degree of non-linearity can be higher than quadratic or even cubic terms can accurately describe. On the other hand, these results also indicate that 
only a negligible number of voxels show the degree of non-linearity more extreme than the fourth exponent or its reciprocal. Inspection of the average $\lambda$-exponents and their kernel densities indicates that the degree of non-linearity increased from FA, RD, AD to MD. Moreover, $\mathrm{MD}$ and $\mathrm{AD}$ showed the largest variability in the $\lambda$-exponent across voxels while FA and RD showed a smaller variance, which was close to 1 . This indicates that the non-linear effects of age on MD and AD are much more heterogeneous. This finding is consistent with previous studies pointing out that the global effect of age on FA is predominantly linear, while the global effect of age on MD was reported to be quadratic (Hsu et al., 2010). Importantly, the slightly leptokurtic distribution across voxels of $\lambda$-exponents for FA reveals that the proportion of all voxels showing a linear association with age is larger than it would be expected based on a Gaussian distribution. This reinforces previous findings on the predominantly linear behavior of FA (Hsu et al., 2010).

Several studies have shown a quadratic relationship between age and diffusion measurements in specific neuroanatomical white matter regions (Hsu et al., 2010; Sala et al., 2012; Westlye et al., 2010). However, previous studies reporting highly non-linear effects were limited to track-based statistics (e.g., Michielse et al., 2010). Hitherto, no study has been able to report voxel-wise statistical evidence for more extreme non-linear associations. In contrast, our results suggest the presence of non-linear effects of different degrees at the level of single voxels and individual neuroanatomical structures, because regression models including Box-Cox transformed measures yielded better fit statistics than the non-transformed measures. In this vein, two aspects of these results deserve further characterization: first, the degree of nonlinearity expressed by the $\lambda$-exponents and their spatial distribution in white matter. The stronger the non-linear effect of age, the farther away is the $\lambda$-exponent from 1 and the larger are the differences in the pace of development across adult age. Therefore, an accurate estimate of the degree of non-linearity is informative about the amount of acceleration or deceleration of age-related changes. Second, the spatial distribution of $\lambda$-exponents reveals not only which white-matter structures show accelerated or decelerated age effects but also the extent to which the voxels in this structure show more or less the same trend in development. This will be discussed in more detail below.

\section{Voxelwise analysis of the $\lambda$-exponent}

The spatial distribution of voxels revealing significant age-related effects was highly similar in both the transformed and the nontransformed model (Figs. 3 and 4). This is due to the fact that for the majority of the voxels, non-linear transformations of FA, MD, AD, and RD do not improve the model fit in comparison to untransformed data. Regarding FA, both transformed and non-transformed models revealed age-related effects in the cortico-spinal tract, optic radiation the splenium of the corpus callosum, small medial parts of the uncinate fascicle, the superior longitudinal fasciculus as well as the inferior frontooccipital fascicle when encompassing the external/extreme capsule system (EC/EmC), bilaterally. Regarding MD, both transformed and nontransformed models revealed age-related effects in the body of corpus callosum, cortico-spinal tract, the frontal white matter, and the superior longitudinal fascicle (in the right hemisphere extending into more dor$\mathrm{sal} /$ parietal regions), bilaterally, as well as in the EC/EmC system, bilaterally. Regarding $A D$, both transformed and non-transformed models revealed age-related effects in the body of the corpus callosum, bilaterally, as well as the right corticospinal tract and the superior longitudinal fasciculus. Regarding RD, both transformed and non-transformed models revealed age-related effects in the cortico-spinal tract, body of the corpus callosum, superior orbitofrontal fasciculus, optic radiation, the inferior fronto-occipital fascicle, especially when encompassing the external/extreme capsule system (EC/EmC), and the long segment

\section{A}
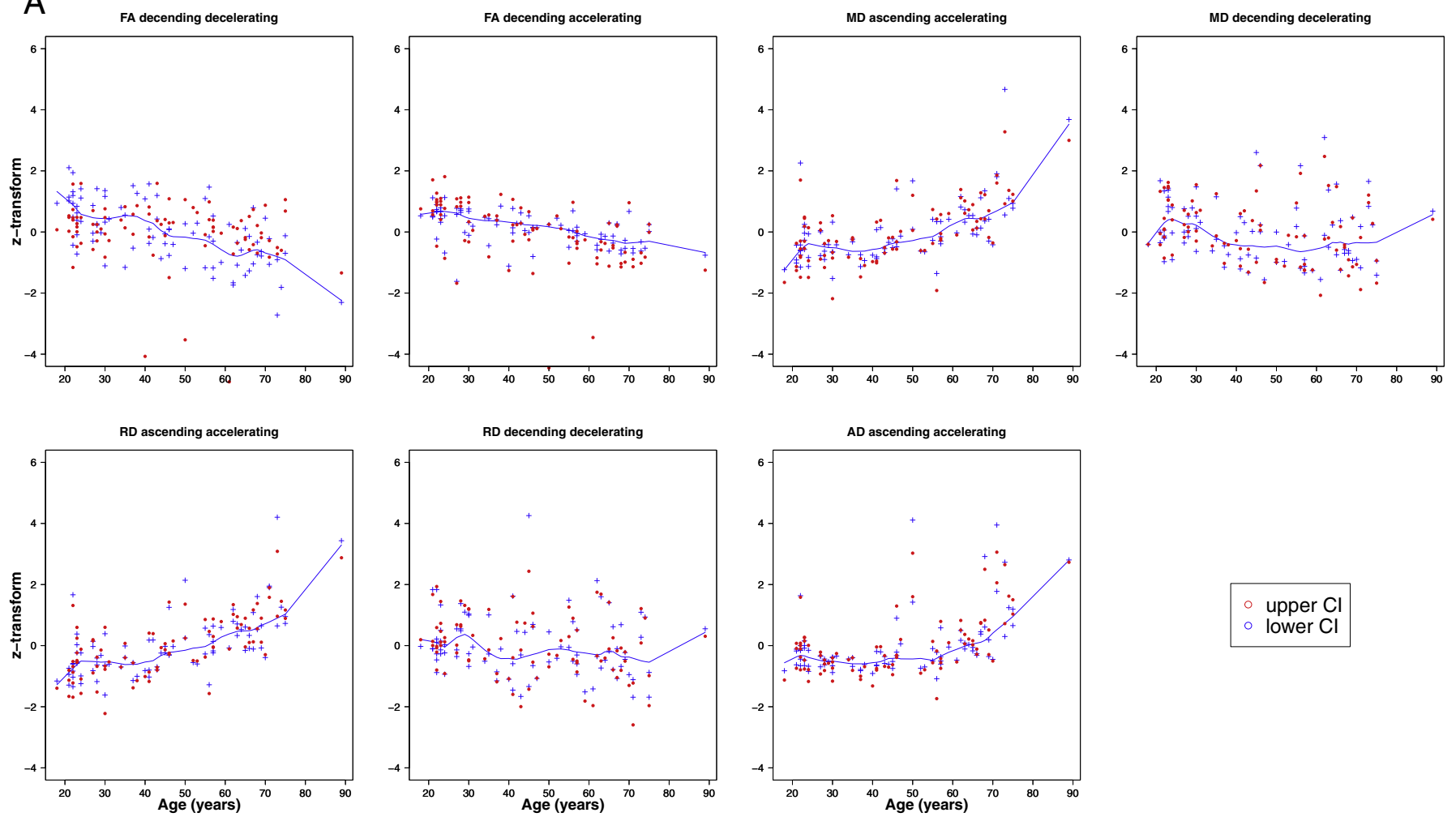

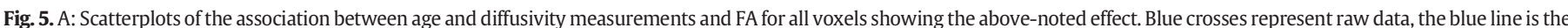

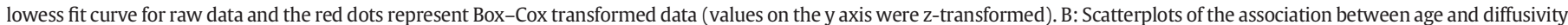

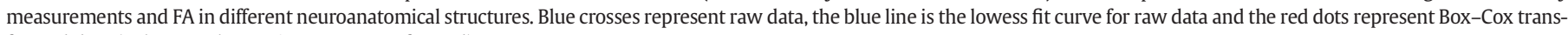
formed data (values on the y axis were z-transformed). 
B
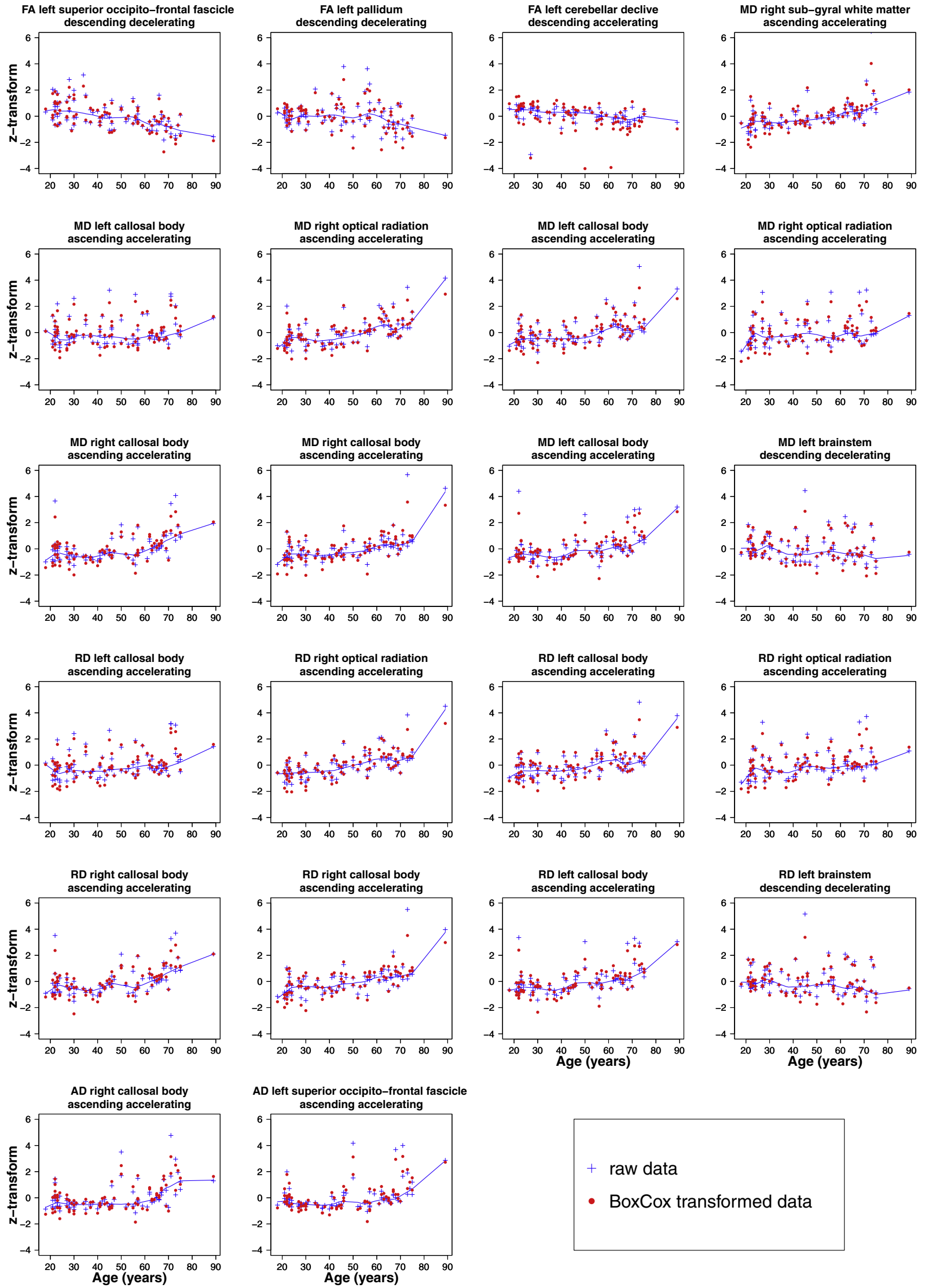

$A D$ left superior occipito-frontal fascicle

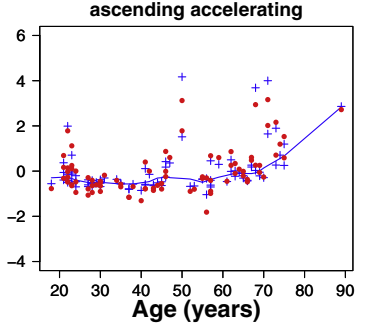

+ raw data

- BoxCox transformed data

Fig. 5 (continued). 

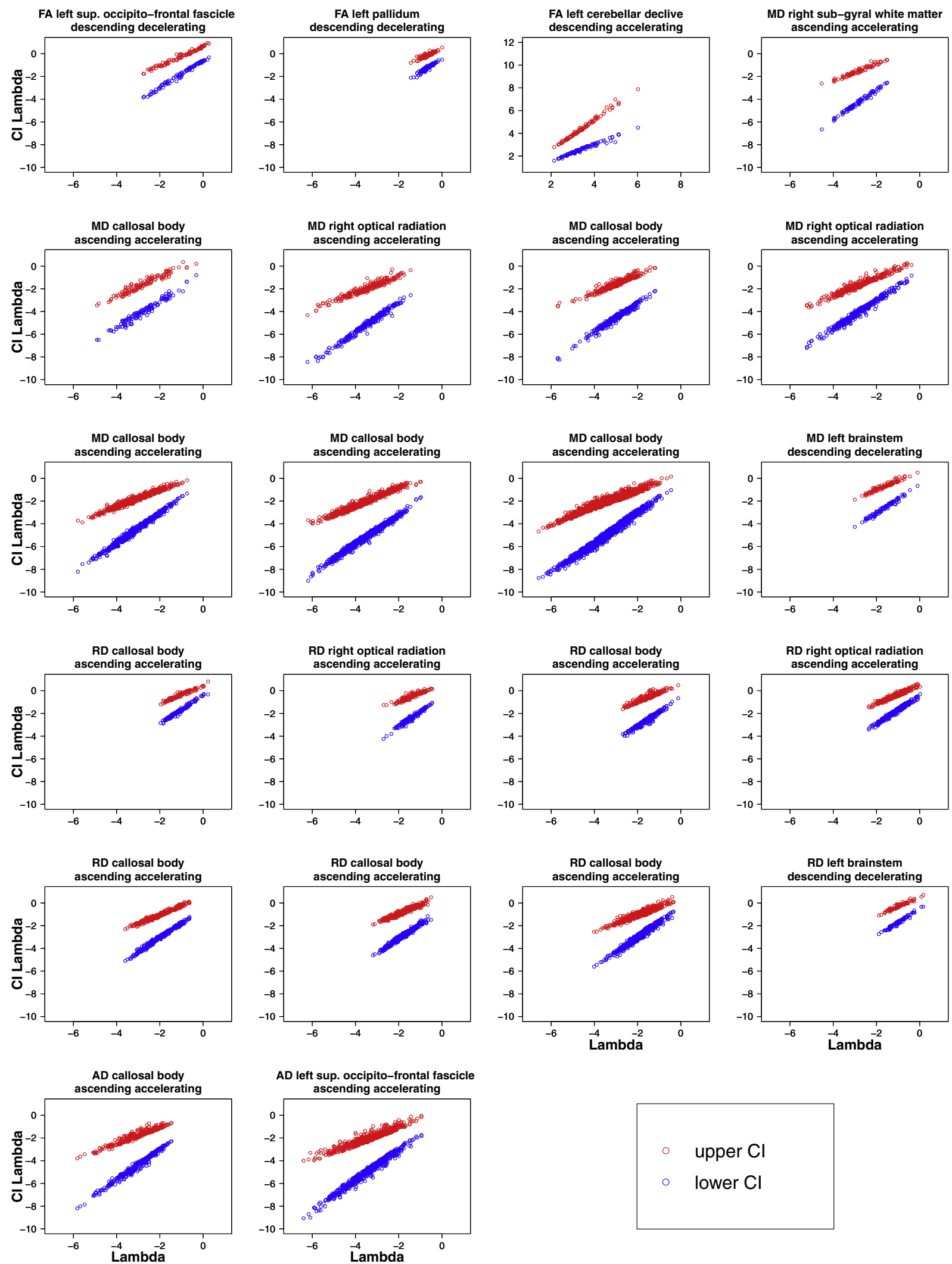

Fig. 6. Confidence intervals in dependence of the $\lambda$-exponents are depicted for all clusters showing significant non-linear age-effects.

of the arcuate fascicule, frontal white matter, the right superior orbitofrontal fasciculus, bilaterally.

Moreover, in a proportion of $0.2 \%$ to $2.8 \%$ of all voxels sensitive to age-effects, the transformed model showed a better fit than the nontransformed model. These results are consistent with previous findings by Hsu et al. (2010), who found a contribution of non-linear regression components only in a small minority of all voxels.

The direct comparison of both models revealed that several neuroanatomical structures are under non-linear influence of age. These results are generally in line with the literature. The transformed model 
Table 4

Location and coordinates of the center of gravity for all clusters showing a significantly better fit for the non-linear model compared to the linear model.

\begin{tabular}{|c|c|c|c|c|c|c|}
\hline Parameter & Effect & Voxels & $\begin{array}{l}\operatorname{COG} X \\
(\mathrm{~mm})\end{array}$ & $\begin{array}{l}\text { COG Y } \\
(\mathrm{mm})\end{array}$ & $\begin{array}{l}\text { COG Z } \\
(\mathrm{mm})\end{array}$ & Location \\
\hline \multirow[t]{3}{*}{ FA } & Descending accelerating & 93 & -21 & -46.4 & -33.2 & Left cerebellar declive \\
\hline & Descending decelerating & 106 & -17 & 2 & -0.2 & Left pallidum \\
\hline & & 98 & -21 & 25 & 8.8 & Left superior occipito-frontal fascicle \\
\hline \multirow[t]{9}{*}{ MD } & Descending decelerating & 97 & -0.9 & -26.7 & -16.9 & Left brainstem \\
\hline & Ascending accelerating & 957 & -21 & -7 & 32 & Left callosal body \\
\hline & & 882 & 23.9 & -39.2 & 34 & Right callosal body \\
\hline & & 513 & 20 & 9 & 29 & Right callosal body \\
\hline & & 322 & 27.3 & -67.8 & 10 & Right optical radiation \\
\hline & & 314 & -28 & -54.8 & 22.3 & Left callosal body \\
\hline & & 269 & 36.5 & -47.6 & 24.7 & Right optical radiation \\
\hline & & 128 & -27 & -65.1 & 11.5 & Left callosal body \\
\hline & & 91 & 31.5 & 8 & 22.3 & Right sub-gyral white matter \\
\hline \multirow[t]{8}{*}{$\mathrm{RD}$} & Descending decelerating & 90 & -1.7 & -27.3 & -17.1 & Left brainstem \\
\hline & Ascending accelerating & 522 & -20 & -4 & 32 & Left callosal body \\
\hline & & 504 & 22.7 & -39.9 & 36 & Right callosal body \\
\hline & & 293 & 19.6 & 8 & 29 & Right callosal body \\
\hline & & 277 & 26.5 & -69.9 & 9.4 & Right optical radiation \\
\hline & & 210 & -29 & -55 & 21.6 & Left callosal body \\
\hline & & 149 & 34 & -51.8 & 23.2 & Right optical radiation \\
\hline & & 123 & -29 & -63.8 & 11.2 & Left callosal body \\
\hline \multirow[t]{2}{*}{$\mathrm{AD}$} & Ascending accelerating & 593 & -21 & -4 & 30 & Left superior occipito-frontal fascicle \\
\hline & & 297 & 20.6 & 7 & 27.9 & Right callosal body \\
\hline
\end{tabular}

revealed significant non-linear age-related effects on FA in the left superior orbitofrontal fasciculus, the left brainstem, and the left cerebellar declive. With the single exception of the left cerebellar declive, significant non-linear effects of age showed consistently a $\lambda$-exponent $<1$ in all diffusivity measures and FA in different white matter structures. We also observed an age-related decrease in FA in the left frontooccipital fascicle and left pallidum. Lebel et al. (2012)) and Bendlin et al. (2010) found an increase in MD in the superior fronto-occipital fascicle. Moreover, Walhovd et al. $(2005,2011)$ observed reductions in the white matter volumes in the left pallidum in function of age.

We also found an increase of MD, RD, and $\mathrm{AD}$ in regions compatible with the localization of fibers from the right and left corpus callosum. Cross-sectional (Lebel et al., 2010; Sala et al., 2012) as well as longitudinal studies (Ly et al., 2014) have reported loss of white matter integrity in these regions. Moreover, Lockhart and DeCarli (2014) and Sullivan et al. $(2001,2008)$ suggested white matter integrity in these regions to be associated with age-related changes in cognitive performance. An acceleration in the increase of MD and RD in function of age was observed in the optic radiation in the present study. These results complement previous findings reporting a negative correlation between age and FA in the optic radiation (Zhang et al., 2014). Finally, age-related effects on the volume of white matter in the cerebellum (Walhovd et al., 2005,2011 ) are consistent with the presence of age-related effects in FA, MD and RD, as found in the present study. In summary, the neuroanatomical structures found to be under non-linear age-related effects in the present study have been reported in several other studies as well, which not only investigated FA and diffusivity measurements, but also the white matter volumes of these structures.

Age-related effects on RD were found in the right optic radiation and the right temporal white matter and finally, age-related effects on RD in the left superior orbitofrontal fasciculus, the right cingulum, and in the left parietal white matter. Non-linear effects of age have been reported in all these white-matter structures in previous studies (Lebel et al., 2012; Sala et al., 2012; Westlye et al., 2010). The present results extend previous ones by approximating the degree of non-linearity by means of the $\lambda$-exponents with higher accuracy. As discussed above, this approach revealed a considerable number of voxels in which even a cubic term in a polynomial regression model would still fail to describe the correct degree of non-linearity. In contrast, the Box-Cox analysis provides a natural and intuitive way to achieve an estimation of the degree of non-linearity.

\section{Accelerating vs. decelerating effects}

Accelerating age-related effects have been observed in FA, MD, RD, and $\mathrm{AD}$. These accelerating age-related effects led FA values to decrease at a faster pace in late adulthood, and $\mathrm{MD}, \mathrm{RD}$, and $\mathrm{AD}$ values to increase at a faster pace in late adulthood. This is an interesting finding, as different studies indicate the presence of aging effects that tend to accelerate in older ages (Hsu et al., 2010; Westlye et al., 2010). In contrast, the agerelated effects observed in $\mathrm{MD}, \mathrm{RD}$, and $\mathrm{AD}$ were accelerating and positive. This reflects larger changes in $\mathrm{MD}, \mathrm{RD}$, and $\mathrm{AD}$ in later rather than in early adulthood. These results are in line with a recent longitudinal study reporting an acceleration of age-related effects to begin in the fifth decade (Sexton et al., 2014).

Importantly, decelerating age-related effects were observed in FA in the left pallidum and left superior fronto-occipital fascicle, and in both $\mathrm{MD}$ and RD in the left brainstem. While the interpretation of accelerating effects is straightforward and reflects the cumulative effect of different aging processes, decelerating age-related effects are more difficult to conceptualize. It seems implausible to us that the speed of aging might genuinely slow down in late adulthood. A more tangible explanation for the deceleration of age-related effects is the result of the selection of participants with sufficient levels of cognitive and brain health to participate in imaging studies. Since the proportion of older individuals from the general population failing to fulfill the typical inclusion criteria for neuroimaging studies is generally larger than the proportion of younger individuals, the shape of deceleration may be observed, which is due only to the selection criteria employed to construct the sample. Further studies combining older individuals with lower levels of cognitive performance and stronger decline of neuroanatomical structures may help to evaluate this claim. In summary, the Box-Cox analysis revealed that diffusion measurements and FA are sensitive to age-effects not all in the same way, since different $\lambda$ coefficients for each diffusivity measurement were observed in different voxels spatially close to each other.

\section{Study limitations and future perspectives}

Since the $\lambda$-exponent can assume any real value, the Box-Cox transformations come closer to the genuine degree of compression presented by natural data than higher-order polynomials, which rely upon a priori 
defined quadratic or cubic trends. However, the Box-Cox analysis is not a method to determine the correct model to fit the data but rather a technique to approximate it with a degree of parsimony higher than most alternative methods. The Box-Cox analysis captures only monotonic non-linear behavior. Therefore, one may argue that age-related effects may be overseen when employing the Box-Cox analysis. Inspection of scatterplots depicting regression slopes for non-linear effects of age on diffusivity as published in the last 15 years (e.g., Hsu et al., 2010; Lebel et al., 2012; Sala et al., 2012) does not reveal any neuroanatomical region in which a monotonic transformation would have behaved much worse than a higher order polynomial regression model, at least not when the analysis is limited to adult age. For this reason, we believe that the Box-Cox analysis can be very useful to detect and describe non-linear effects of age even with the limitation imposed by monotonicity. Finally, application of the Box-Cox analysis to wholebrain data yields a sensitive measurement of the degree of nonlinearity. This can complement evidence obtained for isolated fiber tracts, since the voxel-based Box-Cox analysis does not have to assume that a fiber tract in its entirety responds uniformly to aging. Further studies are required to investigate the heterogeneity of $\lambda$ within the main fiber tracts.

\section{Acknowledgments}

GW was supported by a FWF grant (P 22577-B18), EK by the LeibnizCompetition Fund (SAW-2014-IWM-4) as well as the Margarete von-Wrangell Fellowship of the Ministry of Science, Research and Arts Baden-Wuerttemberg and the European Social Fonds (ESF).

\section{Appendix A. Supplementary data}

Supplementary data to this article can be found online at http://dx. doi.org/10.1016/j.neuroimage.2015.08.003.

\section{References}

Abe, O., Yamasue, H., Aoki, S., Suga, M., Yamada, H., Kasai, K., Masutani, Y., Kato, N., Ohtomo, K., 2008. Aging in the CNS: comparison of gray/white matter volume and diffusion tensor data. Neurobiol. Aging 29, 102-116.

Assaf, Y., Pasternak, O., 2008. Diffusion tensor imaging (DTI)-based white matter mapping in brain research: a review. J. Mol. Neurosci. 34 (1), 51-61.

Bendlin, B.B., Fitzgerald, M.E., Ries, M.L., Xu, G., Kastman, E.K., Thiel, B.W., et al., 2010. White matter in aging and cognition: a cross-sectional study of microstructure in adults aged eighteen to eighty-three. Dev. Neuropsychol. 35 (3), 257-277.

Benjamini, Y., Yekutieli, D., 2001. The control of the false discovery rate in multiple testing under dependency. Ann. Stat. 29, 1165-1188.

Box, G.E., Cox, D.R., 1964. An analysis of transformations. J. R. Stat. Soc. Ser. B 26, 211-252.

Burnham, K.P., Anderson, D.R., 2002. Model Selection and Inference: A Practical Information-theoretic Approach. second ed. Springer, New York.

Carmichael, O., Chen, J., Paul, D., Peng, J., 2013. Diffusion tensor smoothing through weighted Karcher means. Electron. J. Stat. 7, 1913.

Eickhoff, S.B., Stephan, K.E., Mohlberg, H., Grefkes, C., Fink, G.R., Amunts, K., Zilles, K., 2005. A new SPM toolbox for combining probabilistic cytoarchitectonic maps and functional imaging data. NeuroImage 25, 1325-1335.

Eickhoff, S.B., Heim, S., Zilles, K., Amunts, K., 2006. Testing anatomically specified hypotheses in functional imaging using cytoarchitectonic maps. Neurolmage 32 (2), 570-582.

Eickhoff, S.B., Paus, T., Caspers, S., Grosbras, M.H., Evans, A.C., Zilles, K., Amunts, K., 2007. Assignment of functional activations to probabilistic cytoarchitectonic areas revisited. Neurolmage 36 (3), 511-521.

Fjell, A.M., Walhovd, K.B., Westlye, L.T., Østby, Y., Tamnes, C.K., Jernigan, T.L., ..., Dale, A.M., 2010. When does brain aging accelerate? Dangers of quadratic fits in cross-sectional studies. Neurolmage 50 (4), 1376-1383.

Folstein, M.F., Folstein, S.E., McHugh, P.R., 1975. Mini-mental state examination. A practical method for grading the state of patients for the clinician. J. Psychiatr. Res. 12, 189-198.

Gong, N.J., Wong, C.S., Chan, C.C., Leung, L.M., Chu, Y.C., 2014. Aging in deep gray matter and white matter revealed by diffusional kurtosis imaging. Neurobiol. Aging 35, 2203-2216.

Hampel, F.R., Ronchetti, E.M., Rousseeuw, P.J., Stahel, W.A., 1986. Robust Statistics: The Approach Based on Influence Functions. Wiley.

Hautzinger, M., Bailer, M., Hofmeister, D., Keller, F., 2012. Allgemeine Depressionsskala (ADS). Manual. second ed. Hogrefe, Göttingen.

Hsu, J.L., Leemans, A., Bai, C.H., Lee, C.H., Tsai, Y.F., Chiu, H.C., Chen, W.H., 2008. Gender differences and age-related white matter changes of the human brain: a diffusion tensor imaging study. Neurolmage 39, 566-577.
Hsu, J.L., Van Hecke, W., Bai, C.H., Lee, C.H., Tsai, Y.F., Chiu, H.C., Jaw, F.S., Hsu, C.Y., Leu, J.G., Chen, W.H., Leemans, A., 2010. Microstructural white matter changes in normal aging: a diffusion tensor imaging study with higher-order polynomial regression models. NeuroImage 49, 32-43.

Jones, D.K., Symms, M.R., Cercignani, M., Howard, R.J., 2005. The effect of filter size on VBM analyses of DT-MRI data. Neurolmage 26 (2), 546-554.

Knaus, J., 2013. snowfall: Easier Cluster Computing (Based on Snow). R package version 1.84-6.

Lancaster, J.L., Tordesillas-Gutiérrez, D., Martinez, M., Salinas, F., Evans, A., Zilles, K. Mazziotta, J.C., Fox, P.T., 2007. Bias between MNI and Talairach coordinates analyzed using the ICBM-152 brain template. Hum. Brain Mapp. 28 (11), 1194-1205.

Lebel, C., Caverhill-Godkewitsch, S., Beaulieu, C., 2010. Age-related regional variations of the corpus callosum identified by diffusion tensor tractography. NeuroImage 52, 20-31.

Lebel, C., Gee, M., Camicioli, R., Wieler, M., Martin, W., Beaulieu, C., 2012. Diffusion tensor imaging of white matter tract evolution over the lifespan. Neurolmage 60, 340-352.

Lockhart, S.N., DeCarli, C., 2014. Structural imaging measures of brain aging. Neuropsychol. Rev. 24, 271-289.

Ly, M., Canu, E., Xu, G., Oh, J., McLaren, D.G., Dowling, N.M., Alexander, A.L., Sager, M.A Johnson, S.C., Bendlin, B.B., 2014. Midlife measurements of white matter microstructure predict subsequent regional white matter atrophy in healthy adults. Hum. Brain Mapp. 35, 2044-2054

Michielse, S., Coupland, N., Camicioli, R., Carter, R., Seres, P., Sabino, J., Malykhin, N., 2010 Selective effects of aging on brain white matter microstructure: a diffusion tensor imaging tractography study. Neurolmage 52, 1190-1201.

Miranda, M.F., Zhu, H., Ibrahim, J.G., 2013. Bayesian spatial transformation models with applications in neuroimaging data. Biometrics 69 (4), 1074-1083.

Muschelli III, J., 2014. brainR: helper functions to misc3d and rgl packages for brain imaging. R Package Version 1.2.

Nusbaum, A.O., Tang, C.Y., Buchsbaum, M.S., Wei, T.C., Atlas, S.W., 2001. Regional and global changes in cerebral diffusion with normal aging. Am. J. Neuroradiol. 22, 136-142.

Pajevic, S., 2011. Statistical issues in diffusion tensor MRI. Diffus. MRI Theory Methods Appl. 331.

Pfefferbaum, A., Sullivan, E.V., Hedehus, M., Lim, K.O., Adalsteinsson, E., Moseley, M., 2000 Age-related decline in brain white matter anisotropy measured with spatially corrected echo-planar diffusion tensor imaging. Magn. Reson. Med. 44, 259-268.

Pfefferbaum, A., Rohlfing, T., Rosenbloom, M.J., Chu, W., Colrain, I.M., Sullivan, E.V., 2013. Variation in longitudinal trajectories of regional brain volumes of healthy men and women (ages 10 to 85 years) measured with atlas-based parcellation of MRI. NeuroImage 65, 176-193.

Royall, R.M., 1997. Statistical Evidence: A Likelihood Paradigm. Chapman and Hall, New York.

Sakia, R.M., 1992. The Box-Cox transformation technique: a review. Statistician 41, 169-178.

Sala, S., Agosta, F., Pagani, E., Copetti, M., Comi, G., Filippi, M., 2012. Microstructural chang es and atrophy in brain white matter tracts with aging. Neurobiol. Aging 33, 488-498.

Salat, D.H., Tuch, D.S., Greve, D.N., Van Der Kouwe, A.J.W., Hevelone, N.D., Zaleta, A.K. Rosen, B.R., Fischl, B., Corkin, S., Rosas, H.D., Dale, A.M., 2005. Age-related alterations in white matter microstructure measured by diffusion tensor imaging. Neurobiol Aging 26, 1215-1227.

Sexton, C.E., Walhovd, K.B., Storsve, A.B., Tamnes, C.K., Westlye, L.T., Johansen-Berg, H., Fjell, A.M., 2014. Accelerated changes in white matter microstructure during aging: a longitudinal diffusion tensor imaging study. J. Neurosci. 34, 15425-15436.

Smith, S.M., 2002. Fast robust automated brain extraction. Hum. Brain Mapp. 17 (3), $143-155$.

Sullivan, E.V., Adalsteinsson, E., Hedehus, M., Ju, C., Moseley, M., Lim, K.O., Pfefferbaum, A., 2001. Equivalent disruption of regional white matter microstructure in ageing healthy men and women. Neuroreport 12, 99-104.

Sullivan, E.V., Rohlfing, T., Pfefferbaum, A., 2008. Quantitative fiber tracking of lateral and interhemispheric white matter systems in normal aging: relations to timed performance. Neurobiol. Aging 31, 464-481.

Venables, W.N., Ripley, B.D., 2002. Modern Applied Statistics with S. fourth ed. Springer, New York.

Viswanath, V., Fletcher, E., Singh, B., Smith, N., Paul, D., Peng, J., ..., Carmichael, O., 2012 Impact of DTI smoothing on the study of brain aging. Engineering in Medicine and Biology Society (EMBC), 2012 Annual International Conference of the IEEE. IEEE pp. 94-97 (August).

Walhovd, K.B., Fjell, A.M., Reinvang, I., Lundervold, A., Dale, A.M., Eilertsen, D.E., Quinn, B. Salat, D., Makris, N., Fischl, B., 2005. Effects of age on volumes of cortex, white matter and subcortical structures. Neurobiol. Aging 26 (9), 1261-1270.

Walhovd, K.B., Westlye, L.T., Amlien, I., Espeseth, T., Reinvang, I., Raz, N., Agartz, I., Salat, D., Greve, D., Fischl, B., Dale, A., Fjell, A.M., 2011. Consistent neuroanatomical age-related volume differences across multiple samples. Neurobiol. Aging 32 (5), 916-932.

Westlye, L.T., Walhovd, K.B., Dale, A.M., Bjørnerud, A., Due-Tønnessen, P., Engvig, A., Grydeland, H., Tamnes, C.K., Østby, Y., Fjell, A.M., 2010. Life-span changes of the human brain white matter: diffusion tensor imaging (DTI) and volumetry. Cereb. Cortex 20, 2055-2068.

Whitcher, B., Schmid, V.J., Thornton, A., 2011. Working with the DICOM and NIfTI Data Standards in R. J. Stat. Softw. 44 (6), 1-28.

Zhang, X., Li, B., Shan, B., 2014. Age-related white matter degradation rule of normal human brain: the evidence from diffusion tensor magnetic resonance imaging. Chin. Med. J. 127, 532-537.

Ziegler, G., Ridgway, G.R., Dahnke, R., Gaser, C., Alzheimer's Disease Neuroimaging Initiative, 2014. Individualized Gaussian process-based prediction and detection of local and global gray matter abnormalities in elderly subjects. NeuroImage 97 , 333-348. 